\title{
Design of ITS Architecture and Implementation of Safety Applications Based on the WAVE Protocol Stack
}

\author{
Yu Wang, Zhizhong Ding, Xue xia, Zhentao Li and Huijing Shi
}

\begin{abstract}
Intelligent Transportation System (ITS) has become a hot topic in recent years. The Wireless Access in Vehicular Environment (WAVE) protocol stack is a set of standards intended to provide safety, seamless and effective communication for vehicle-to-vehicle (V2V) or vehicle-to-infrastructure (V2I). It has been the center of major attention in the research field of the ITS. In this paper, after present a fairly detailed description of the WAVE protocol stack, we design a system architecture of ITS based on the WAVE and implement two safety applications: Emergency Electronic Brake Lights and Intersection Collision Warning which are specified in the SAE J2735 standard. We also develop a simulation program and performed field measurement. The result shows that the architecture can provide wireless communication in a vehicular environment with low-latency and the Safety applications run successfully using our algorithm.
\end{abstract}

Index Terms-WAVE, ITS, vehicle safety applications, IEEE 802.11p, IEEE 1609.

\section{INTRODUCTION}

With the rapid development of the automobile industry, the traffic congestion and safety problems have been increasingly serious. Statistic suggests that drivers spend about 101 hours in congestion in London 2015, according to Intrix which is a traffic data analysis company. To overcome these problems, researchers are increasingly interested in the Intelligent Transportation System and the wireless communication technology used in the ITS [1].

Some researchers put forward the Device to Device (D2D) based on the $5 \mathrm{G}$. But the $5 \mathrm{G}$ standard is still in its primary stage The EU announced that they would provide the $5 \mathrm{G}$ service until 2025 in the proposal called "5G for Europe: An Action Plan". And the device must send the Session Initiation Protocol (SIP) to the base station before D2D communication [2][3]. It would increase the pressure on the base station.

IEEE completed the WAVE protocol stack in 2010. It consists of the SAE J2735 standard which defines the application layer and IEEE 1609 standards and IEEE 802.11p

Manuscript received December 19, 2016

This work has been supported by the "VANET system" of 2015 HFUT Qiu Shi Plan (ID: JZ2015QSJH0536). Sponsored and financial supported by the ECTHF in Hefei.

$\mathrm{Yu}$ Wang is with School of Computer and Information, Hefei University of Technology, Hefei, China (e-mail: 18010877000@163.com).

Zhizhong Ding is with School of Computer and Information, Hefei University of Technology, Hefei, China (e-mail: zzding@hfut.edu.cn).

Xue Xia and Zhentao Li is with School of Computer and Information, Hefei University of Technology, Hefei, China.

Huijing Shi is with Kunming Shipbrone equipment research and testing center which is the modification of the IEEE 802.11 [4]. The WAVE protocol stack supports safety and low latency transactions among vehicles (V2V), or between vehicles and infrastructure components (V2I). And the WAVE devices can implement Ad-Hoc networking by playing the role of "provider" or "user" [5].

However, there is no mature commercial system based on the WAVE. Some companies like ARADA and Cohda have just produced the network module with 802.11p and achieved 1609 protocols. But there is no research on the ITS architecture and implementation of the application layer based on the WAVE.

Therefore, we want to design an Intelligent Transportation System which meets the need for data exchange in a high-speed environment based on the WAVE and program the safety applications. This system is able to relieve the traffic pressure, prevent the traffic accident and follow the trend of mobile internet.

In this paper, we take an overview of the WAVE protocol stack at the first. Then submit the design of the overall system architecture including the topological structure of the system and the deployment of the roadside RSU. We design two channel allocation methods for normal transmission and video data transmission and achieve time synchronization based on the algorithm in the Network Time Protocol (NTP). The program architecture in the application layer is detailed in the next. Then, we improve the judgment method in the SAE J2735 and put forward the algorithms for the safety application with existing sensor like GPS in the Chapter IV. Simulation with the $\mathrm{C \#}$ application that we programmed and field measurement are at the end of this paper.

\section{OVERVIEW OF THE WIRELESS ACCESS IN VEHICULAR ENVIRONMENT}

The U.S. Federal Communications Commission (FCC) have allocated $75 \mathrm{MHz}$ of licensed spectrum in the $5.9 \mathrm{GHz}$ (from $5.850 \mathrm{GHz}$ to $5.925 \mathrm{GHz})$ for Dedicated Short-Range Communication (DSRC) [5]. And the WAVE protocol stack is a set of standards used for wireless communication in the DSRC. 


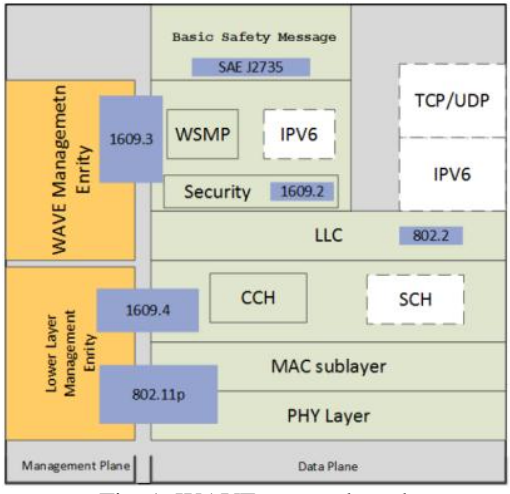

Fig. 1. WAVE protocol stack

Fig. 1 illustrates the architecture of the WAVE protocol stack: IEEE Std 802.11p for the PHY layer and MAC sub- layer, IEEE Std 1609.4 for channel coordination and switching, IEEE Std 1609.3 for networking services and SAE J2735 standard for the application layer [5].

\section{A. IEEE Std $802.11 p$}

The $802.11 \mathrm{p}$ standard is the complement and modification of the IEEE 802.11 standard and specifies the PHY layer function [4]. The PHY has been optimized to support usage by vehicles travelling at highway speed. It introduces a new model of communication-Outside Context of BSS (OCB). A WAVE device is not a member of the BSS and it does not utilize the IEEE 802.11 authentication, association or data confidentiality services when dot11OCBEnabled (a data frame in the MAC layer) is true. So the $802.11 \mathrm{p}$ is particularly well-suited for using in rapidly varying communication environments.

\section{B. IEEE Std 1609.4}

The IEEE Std 1609.4 specifies Channel coordination and consists of addition features for OCB operations in the MAC sub-layer [7]. The CSMA/CA is the traditional solution for channel collision, but it can not guarantee that a node can access the channel in time which is not suitable for security data transmission. So the 1609.4 defines multiple channel access to provide a reliable protection mechanism for transmission. Fig. 2 shows the $5,9 \mathrm{GHz}$ spectrum allocation.

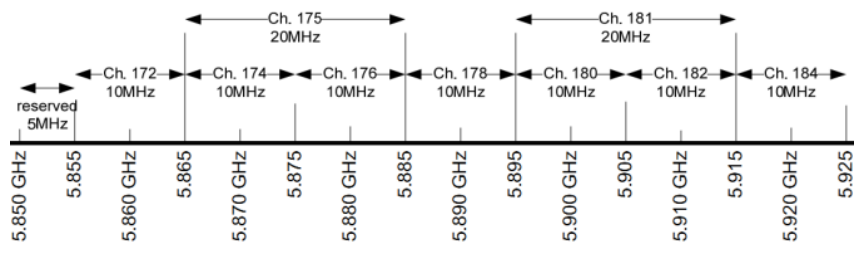

Fig. 2. Spectrum allocation

Channel 178 is the control channel $(\mathrm{CCH})$ and channels 172 , $174,176,180,182,184$ are the service channels $(\mathrm{SCH})$. Channels 174 and 176 and channels 180 and 182 could be combined to produce two twenty-megahertz channels, channels 175 and 181, respectively [5]. The SCH can transmit WSMP and IP packets while the $\mathrm{CCH}$ can only transmit WSMP [5]. Because there are two types of channels, the 1609.4 provides the multi-channel coordination by dividing 1 second into several $\mathrm{SCH}$ intervals and $\mathrm{CCH}$ intervals. The duration of the $\mathrm{CCH}$ and $\mathrm{SCH}$ intervals are stored in the MIB attributes CchInterval and SchInterval [7]. Channel coordination allows a single-PHY device access to high-priority data and management traffic during the $\mathrm{CCH}$ interval, as well as general higher layer traffic during the $\mathrm{SCH}$ interval [7]. The synchronization function is also mentioned in this standard that allows the WAVE devices to perform the channel coordination function.

\section{IEEE Std 1609.3}

WAVE Networking Services is specified in this standard and consists of the data plane and the management plane. The data plane defines two networking protocols: WAVE Short Message Protocol (WSMP) for WAVE Short Message (WSM) data and TCP/IP for IP data [8]. The WSMP is optimised for air interface efficiency and low-latency in support of vehicular application [8]. A WAVE device can control physical parameters (channel number, transmitter power, data rate, etc) directly by the WSMP. The minimum packet overhead for TCP/IP is 48 bytes, whereas for WSMP 5 bytes is enough, and even with options and extensions it rarely exceeds 20 bytes, which is quite valuable for vehicular communications concerning lower latency and higher reliability [9]. The management plane defines the WAVE Management Entity (WME) which performs the management function including receiving and processing request from the high layer entities, monitoring WAVE Service Advertisement (WSA) and maintaining the Management Information Base (MIB) [8]. There are two WAVE device roles defined in the standard. The "provider" role is assumed by a device transmitting WSA indicating its availability for data exchange in the SCH. The user role is assumed by a device monitoring for received WSA, with the potential to participate in the SCH data exchange [5].

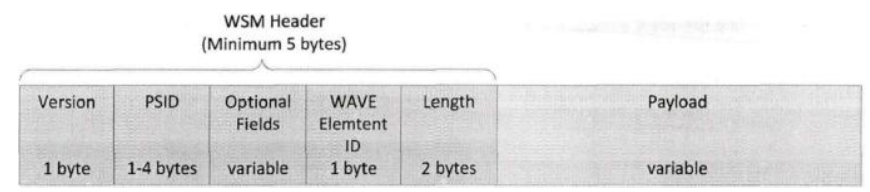

Fig. 3. WAVE Short Message format

\section{SAE J2735}

The SAE J2735 standard is developed by the Society of Automotive Engineers (SAE) in the U.S. and specifies message sets, data frames and elements specifically for use by the application intended to utilise the $5.9 \mathrm{GHz}$ DSRC for WAVE devices. The MSG_BasicSafetyMessage (BSM) is the most important message set used in a variety of security application. This message is broadcast to surrounding vehicles at a rate of every $10 \mathrm{~ms}$ [10]. The following is the ASN.1 format for the BSM:

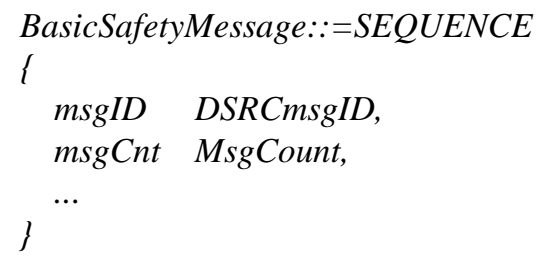

There are also seven safety application scenarios described in 
this standard. We have designed the algorithm with message set and optimised the way of judgment which will be explained in Chapter IV.

\section{OVERALl DESIGN OF THE SYSTEM}

In this chapter, we introduce the design of the ITS system based on the WAVE from five aspects.

\section{A. The topology of the whole system}

The topology of the whole system is shown in Fig. 4.

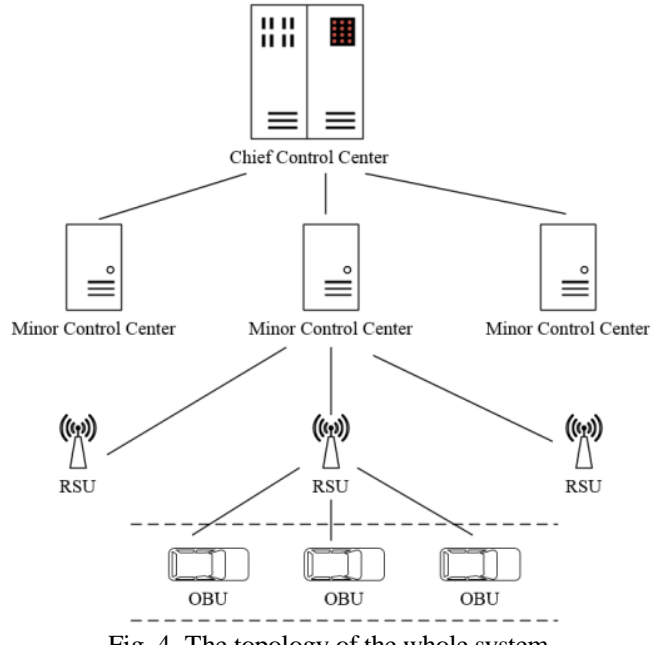

Fig. 4. The topology of the whole system

The WAVE devices can be divided into two types: Road Side Unit (RSU) and On Broad Unit (OBU). The OBU is mounted on the car driving on the road. It communicates with the RSU and other OBU via the WAVE protocol. The roadside RSU provides network access for the OBU and send some important vehicle information to the Minor Control Center through the network cable or "city WIFI". The Chief Control Center collects the traffic data of the entire city then analyses the traffic state and traffic flow. According to the above description, the OBU is like the Station (STA) in the WIFI standard while the OBU and the Minor Control Center are like the Access Point (AP) and Access Controller (AC) [5].

We use the tree topology for the whole system design and the advantages are:

1) It is easy to add new branches and nodes or maintain the devices.

2) the functional design of each layer is distinct. The device is only responsible for the data exchange of its layer. So the function between the layers is "low-coupling".

3) The system architecture is different from the general tree topology. Devices in a tree topology can not continue working when a root node fails. But the WAVE devices can communication with each other by an Ad Hoc network.

\section{B. Roadside RSU deployment}

Fig. 5 shows the deployment of the roadside RSU.

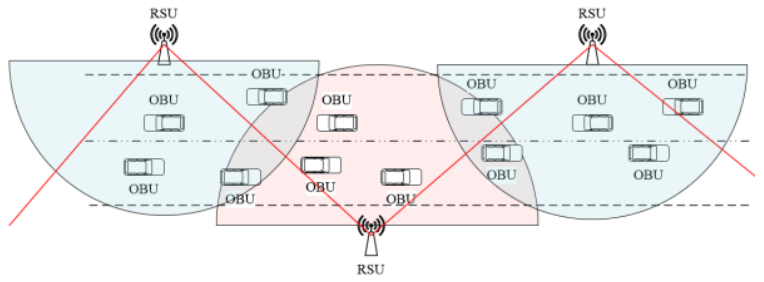

Fig. 5. The deployment of the roadside RSU

The signal coverage is well-distributed by a $\mathrm{Z}$ font deployment. The OBU can improve the location accuracy by calculating its position relationship with the RSUs.

\section{Channel coordination and allocation}

There are different channels for different kinds of message transmission which are introduced in Chapter II. The MAC layer is in charge of channel switching. But the time for switching is unclear for the MAC layer, and it has to be specified by the application layer.

The application layer can control the selection of the channels by WSMP, but developers need to understand the conception and operating mechanisms to construct the efficient allocation mode. The following is the channel allocation scheme we proposed.

TABLE I: CHANNEL ALlOCATION (DEFAULT)

\begin{tabular}{llllll}
\hline \hline \multicolumn{5}{c}{ 1 Second } \\
\hline \hline \multirow{2}{*}{ CCH178 } & SCH172 & CCH178 & SCH175 & CCH178 & $\ldots$ \\
\hline $25 \mathrm{~ms}$ & $25 \mathrm{~ms}$ & $25 \mathrm{~ms}$ & $25 \mathrm{~ms}$ & $25 \mathrm{~ms}$ & $\ldots$ \\
\multirow{2}{*}{ WSMP } & V2V & WSMP & IP & WSMP & $\ldots$ \\
& Security & & Packages & & \\
\hline \hline
\end{tabular}

According to the WAVE protocol, the transmission of 2312 bytes requires $6.5 \mathrm{~ms}$ in the worst case [7]. The transmission rate is approximately $2.84 \mathrm{Mbps}$. Thus, the $\mathrm{CCH}$ allows the access for three nodes $(19.5 \mathrm{~ms})$ at one $\mathrm{CCH}$ interval $(25 \mathrm{~ms})$. And about 60 nodes in one second. Suppose the distance between the RSUs is $400 \mathrm{~m}$, and the speed is about $40 \mathrm{~km} / \mathrm{h}$, then the number of cars (OBUs) is about 50 that exchange safety data with the RSU via $\mathrm{CCH}$. So the default channel allocation can meet the transmission of WSM and IP in the general case.

TABLE II: Channel Allocation (VIDEO TRAnSmission)

\begin{tabular}{llllll}
\hline \hline \multicolumn{5}{c}{ 1 Second } & \\
\hline \hline CCH178 & SCH172 & CCH178 & SCH175 & CCH178 & $\ldots$ \\
\hline $15 \mathrm{~ms}$ & $20 \mathrm{~ms}$ & $15 \mathrm{~ms}$ & $50 \mathrm{~ms}$ & $15 \mathrm{~ms}$ & $\ldots$ \\
\multirow{2}{*}{ WSMP } & V2V & \multirow{2}{*}{ WSMP } & IP & WSMP & $\ldots$ \\
& Security & & Packages & & \\
\hline \hline
\end{tabular}

This channel allocation is appropriate for the transmission of video data when traffic is light. The delay of IP data is $50 \mathrm{~ms}$ $(15 \mathrm{~ms}+20 \mathrm{~ms}+15 \mathrm{~ms})$ at this time. It implies that the accumulation of video data in the time-delay must be transmitted within the IP interval of $50 \mathrm{~ms}$. If the rate of video is $1 \mathrm{Mbps}$, then the allocation scheme meets the requirements of video transmission because the transmission rate is about 2.84Mbps. 


\section{Synchronization}

Synchronize is required after the channel assignment. The nodes which wish to exchange data want each other in this system must access the same channel first. Therefore, only the synchronized devices can transmit security data or user data via the WAVE protocol.

The IEEE 1609.4 mentions the synchronization, but it does not specify the implementation methods. We implement synchronization by obtaining timestamp via GPS and packing it in WSMP. It is modeled the clock synchronization algorithm in the Network Time Protocol (NTP) [11]. The experiment demonstrates that our synchronization error is within $m s$ level, it will be shown in the Chapter V. The following figure is the flowchart of synchronization.

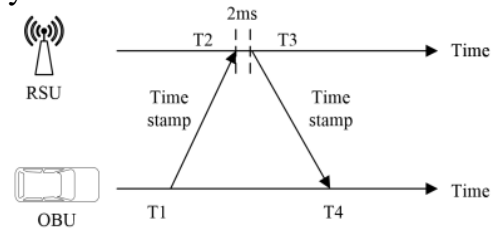

Fig. 6. The flowchart of synchronization

\section{E. Data Transmission}

Data can be transmitted after the channel allocation and the synchronization.

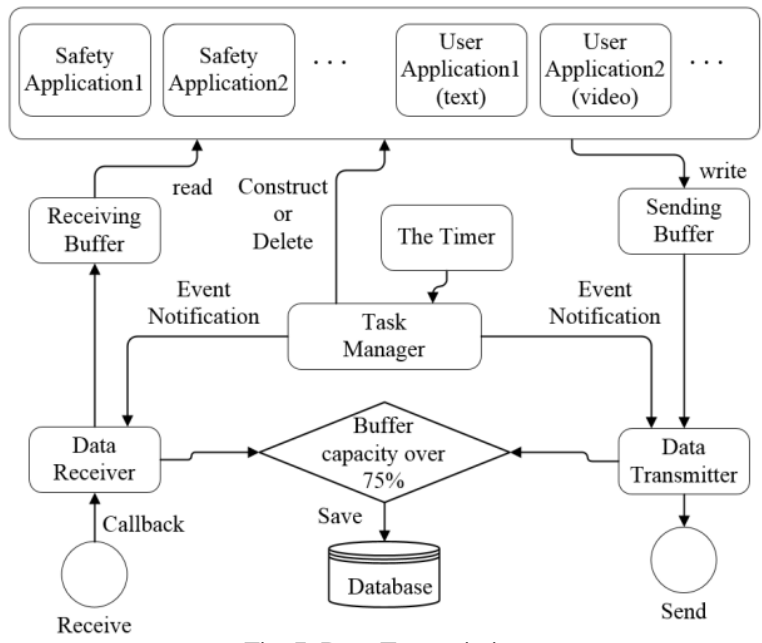

Fig. 7. Data Transmission

Fig. 7. is the flowchart of data transmission. The subapplication sends the data to the corresponding data buffer. Then the Task Manager calls the Data Transmitter to send the safety data and the user data. The receiving process is just the opposite. The Task Manager is in charge of the switching of sub-applications and giving the event notification. The main application saves data to the database when the percentage of buffer capacity is more than $75 \%$.

\section{SAFETY APPLICATIONS}

Improving the driving safety is an important goal for the research of ITS. Thus the major part of the application layer in WAVE devices is the safety applications.

\section{A. Safety Applications Defined in the SAE J2735}

The SAE standard defines seven types of safety applications including: "Intersection Collision Warning", "Emergency Electronic Brake Lights", "Pre-Crash Sensing" and so on. Although the standard mentions that there are "Algorithms" for safety applications, it does not specify how to implement the "Algorithms". Such as how to assess a braking event is dangerous with the BSM broadcast from surrounding cars. And every safety application defined in the standard needs sensors except GPS and other systems like a map database [10].

\section{B. Implementation of two major safety application}

In order to achieve the safety application defined by the standard, we improved the method of judgment and used the existing sensor like GPS for the design of algorithm. The following are two safety applications which have been implemented in the system. We used $\mathrm{C}++$ for programming.

1. Emergency Electronic Brake Lights

When a car is braking in the system, a BSM with the information of braking is sent to surrounding vehicles by the OBU. Surrounding vehicles receive the BSM and judge the risk level by the following algorithm. This safety application increases the transmission distance of braking information and provides some important vehicle information like the value of deceleration. We define the car which is braking and sending the BSM is vehicle " $\mathrm{A}$ " and which receives the BSM is vehicle "B". The safety application is a class file called CObuEmerBrakWarn.cpp in the program.

The running mark of the CObuEmerBrakWarn which named "isRunning" will be set to true when the task manager detected that the speed is higher than $20 \mathrm{~km} / \mathrm{h}$. Then the program will determine the current value of acceleration in the $\mathrm{x}$-axis (we obtained the value by the gyroscope MPU6050). If the value is less than $-0.4 \mathrm{~g}$ which defined in SAE J2735 [10], the program will set the value of EventFlag frame to HardBreakinng in BSM. Fig.8. shows the process.

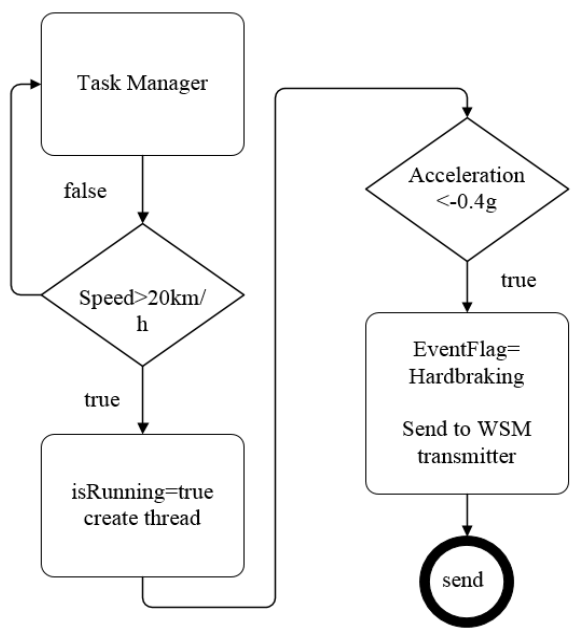

Fig. 8. Braking information transmission

When vehicle "B" receives the BSM message from vehicle " $A$ ", it processes the message and determines that whether it is 
relevant from three steps. We designed a class named CwarnAlg.cpp to implement related algorithms.

First of all, comparing the value of heading in BSM and the value via GPS. Vehicle "A" must be in the opposite lane when the difference is 180 degrees. Through experimentation, the application does further judgment if the difference between heading values is less than 30 degrees.

Second, the program constructs an equation to judge whether vehicle "A" and " $\mathrm{B}$ " are in the same lane according to the location information and the heading value from BSM and GPS. The equation is the moving trajectory equation of vehicle "B":

$$
y-y_{B}=k\left(x-x_{B}\right)
$$

where $x_{B}$ and $y_{B}$ are the position of vehicle "B". $k$ equals the value of heading adds 90 degrees because heading expresses degrees from North [10]. Depending on the location information of vehicle " $\mathrm{A}$ " from the BSM and equation (1), we can calculate the distance of vehicle " $\mathrm{A}$ " and the moving trajectory equation of vehicle " $\mathrm{B}$ ":

$$
\text { Dist }=\frac{\left|k \times x_{A}-y_{A}+y_{B}-k \times x_{B}\right|}{\sqrt{k^{2}+1}}
$$

where Dist is the distance between "A" and "B". $x_{A}$ and $y_{A}$ are the position of vehicle "B". Based on the Road Width Standard [12], program determines vehicle "A" and "B" are in the same lane if the value of Dist is less than $2 \mathrm{~m}$. Fig.9 indicates the determination.

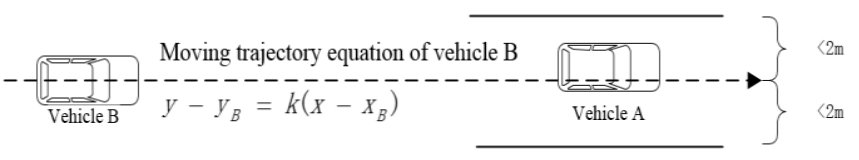

Fig. 9. The vehicles are in the same lane

Finally, the program determines whether vehicle " $\mathrm{A}$ " is in front of vehicle "B" by the following method.

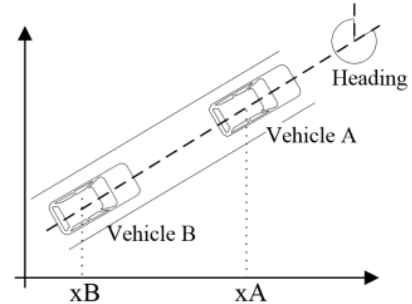

Fig. 10. The position relationship between the vehicle A and $B$

The vehicle with a higher value of $\mathrm{x}$-axis is in front of another vehicle when the heading value is $225^{\circ}$ to $315^{\circ}$ as shown in Fig. 10. In comparison, the vehicle with a lower value of $\mathrm{x}$-axis is in front of another vehicle when the heading value is $45^{\circ}$ to $135^{\circ}$. The judgment of the $\mathrm{Y}$ axis is similar to that.

The program determines that whether the hard braking message is relevant after the three steps above. Then The IsDangBrakLevel() method in the CWarnAlgo determines the risk level.

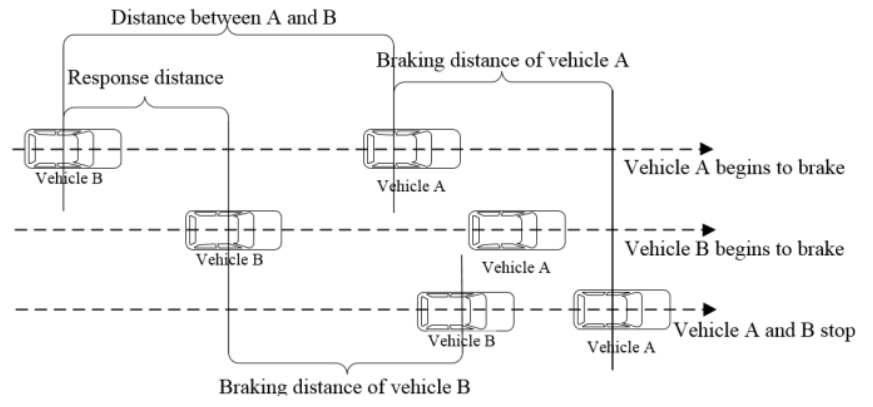

Fig.11. Judgment of braking danger level

According to the figure, equation (3) should be satisfied if vehicle "B" does not collide with vehicle "A" exactly.

$$
L_{R}+S_{B}=L_{A B}+S_{A}
$$

$L_{R}$ is the response distance, we can get it from the speed of vehicle "B" and the time of human brain response and data transmission delay (about $0.5 \mathrm{~s}$ ) [10]. $L_{A B}$ is the distance between vehicle "A" and "B", we can get it from the PositionLocal3D frame in the BSM. $S_{A}$ and $S_{B}$ is the braking distance of "A" and "B", we can calculate it by using $S=V^{2} / 2 \alpha$ and the parameters can be got from the AccelerationSet 4 Way frame in the BSM.

We can calculate vehicle "B" 's deceleration $\alpha_{B}$ by the following equation (4).

$$
\alpha_{B}=\frac{V_{B}^{2}}{2\left(L_{A B}+\frac{V_{A}^{2}}{2 \alpha_{A}}-0.5 V_{B}\right)}
$$

We divided the risk level into three levels. There is a potential danger when $\alpha_{B}<0.4 \mathrm{~g}$, a regular danger when $0.4 \mathrm{~g}<\alpha_{B}<0.75 \mathrm{~g}$, and an emergency situation when $\alpha_{B}>0.75 \mathrm{~g}$. Fig. 12 displays the process of receiving and judgment.

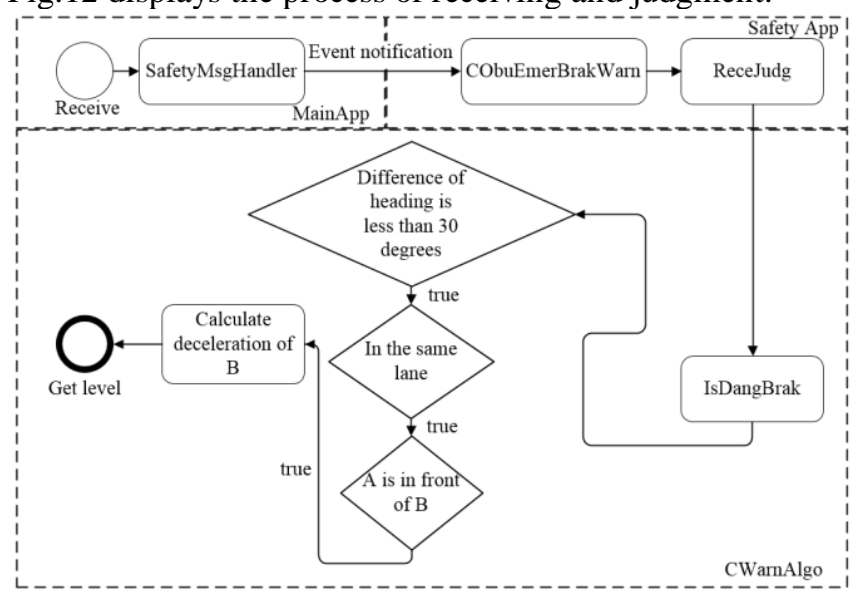

Fig.12. Receiving and handing braking information

\section{Intersection Collision Warning}

We improved the judgment method in the SAE J2735. The position information of the road is obtained from the roadside unit RSU, and the direction perpendicular to the road stop line is taken as the direction of the lane through the road center point. Calculating the direction of the lane angle, and when the difference of the lane angle and the direction of the vehicle 
heading angle is within 30 degrees, the car can be considered in the road and being about to cross the intersection. In this way, the traffic situation on the other lanes will be pushed out and a warning will be issued when there are two or more vehicles coming to the intersection.

\section{Simulation ANd Field Measurement}

We developed our program on the ARADA's WAVE devices (with a Linux kernel 2.6).

\section{A. Simulation}

We programmed a simulation program by $\mathrm{C \#}$ and OpenGL to verify the algorithm in safety application. The WAVE equipment connects to the computer through socket.

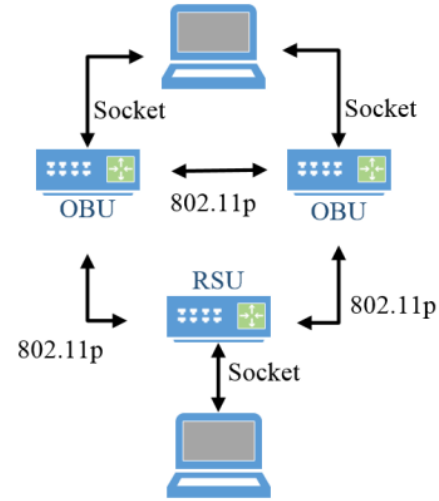

Fig.13. Connection diagram of WAVE devices and PC

First of all, we simulate the communication between the OBUs. The PC generates lots of vehicle information including vehicle's ID, position, heading, acceleration and so on. Then the PC sends the vehicle information data to one OBU via socket. The OBU broadcasts vehicle information in the format of BSM. Another OBU tests the safety application by the receiving dates.

Also, we can simulate the operation of the RSU in the intersection. The RSU runs the Intersection Collision Warning application and maintain the application buffer. Then the RSU sends the buffer data to the PC through socket. Fig.14 shows the simulation program. The vehicle snapshot of the current intersection is saved in the RSU program buffer and displayed in a "listbox". The lower right corner shows the risk level when vehicle " $\mathrm{B}$ " receiving the safety message about vehicle " $\mathrm{A}$ ".

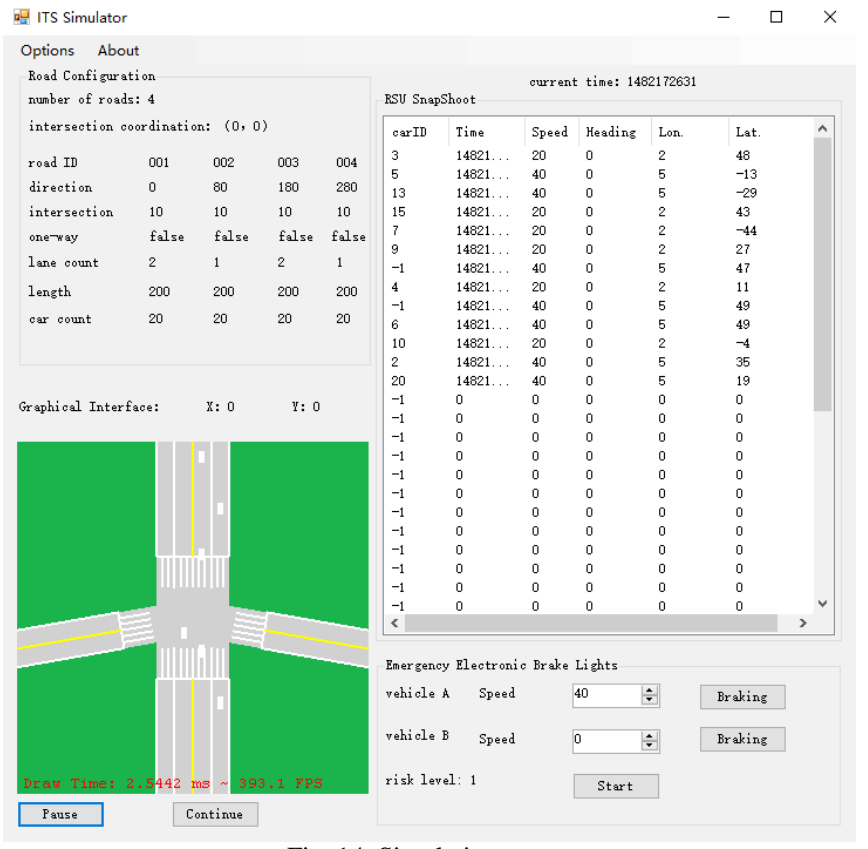

Fig. 14. Simulation program

\section{B. Field Measurement}

We have conducted field measurement too. And the test of time delay, text data exchange is successful as the Fig.15.

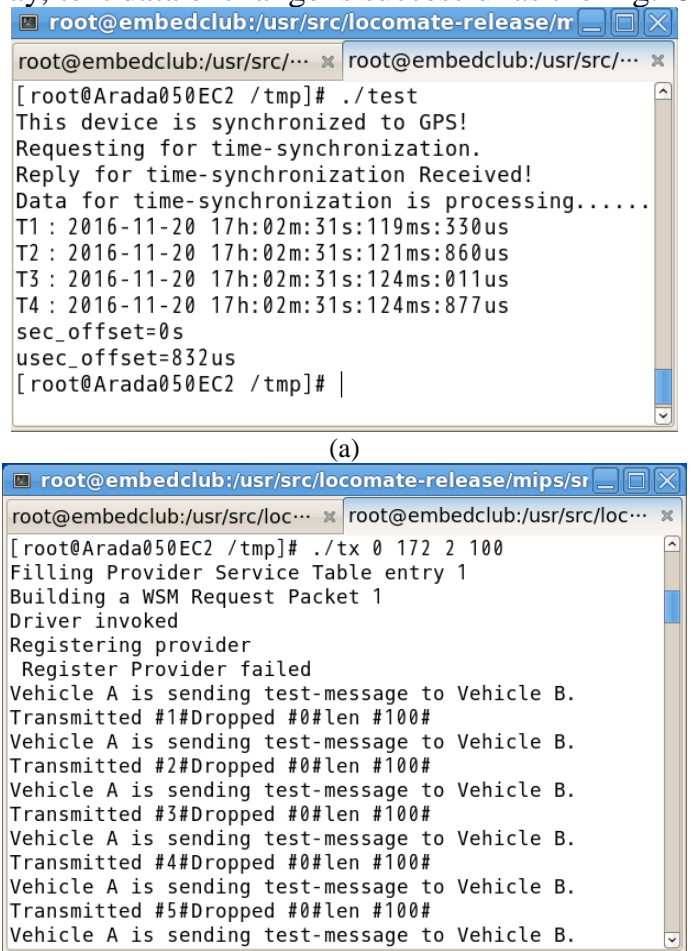

(b) 


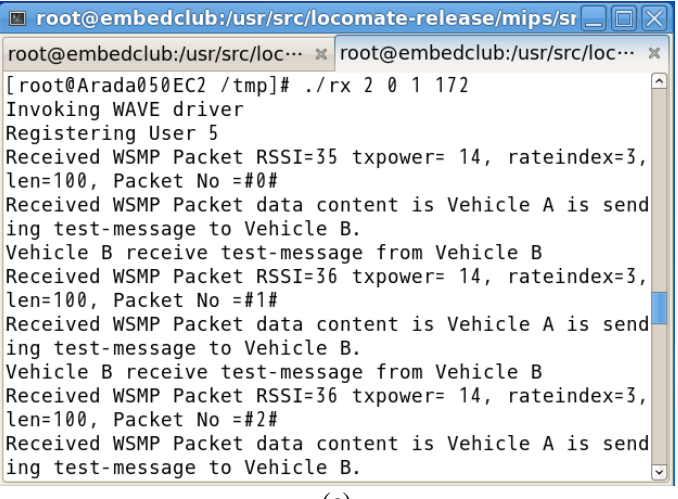

(c)

Fig. 15. Field Measurement

\section{CONCLUSION}

This paper describes the design of an ITS based on the WAVE protocol stack and illustrates the deployment of the WAVE devices, the channel allocation under different conditions and synchronization between the OBU and the RSU. We have implemented two major safety applications and performed simulation and field measurement successful.

For the further work, we will implement the rest of the safety application in the SAE J2735 and try to port our program to an Android development board.

\section{ACKNOWLEDGMENT}

The authors thanks the support from the School of Computer and Information of Hefei University of Technology and Anhui Institute of Quality and Standardization.

\section{REFERENCES}

[1] The framework for the deployment of Intelligent Transport Systems in the field of road transport and for interfaces with other modes of transport, Official Journal of the European Union, 7 July 2010.

[2] K. Doppler, M. Rinne, C. Wijting, "Device-to-device communication as an underlay to LTE-advanced networks", presented at IEEE Communication Mag, 42-49, 2009.

[3] F. Malandrino, C. Casetti, C. F. Chiasserini, "Toward D2D-enhanced heterogeneous networks", presented at IEEE Communication. Mag., 94-100, 2014.

[4] Wireless LAN Medium Access Control (MAC) and Physical Layer (PHY) Specifications Amendment 6: Wireless Access in vehicular Environments, IEEE Standard, 15 July 2010.

[5] Guide for Wireless Access in Vehicular Environments (WAVE) Architecture, IEEE Standard, 2014.

[6] Wireless Access in vehicular Environments Security Services for Application and Management Message, IEEE Standard, 2013.

[7] Wireless Access in vehicular Environments - Multi-channel Operation, IEEE Standard, 2010.

[8] Wireless Access in vehicular Environments - Networking Services, IEEE Standard, 2010.

[9] Minpeng Miao, Qiang Zheng, Kan Zheng, Zhiwei Zeng, "Implementation and Demostration of WAVE NetWorking Services for Intelligent Transportation System", Internet of Vehicles-Technology and Services (IOV), Beijing China, Sept 1-3,2014, 130-139.

[10] Draft SAE J2735 Dedicated Short Range Communications (DSRC) Message Set Dictionary, SAE Standard, 2008.

[11] David L. Mills, "Computer Network Time Synchronization: The Network Time Protocol", 12 December, 2010.

[12] "Lane Width: Chapter 3: The 13 Controlling Criteria. US Department of Transportation Federal Highway Administration", Retrieved June 3, 2013.

[13] Martinez, F.J., Chai-Keong, Emergency Services in Future Intelligent Trans-portation Systems Based on Vehicular Communication Networks. IEEE Intelligent Transportation Systems Magazine, 6-20, 2010.
[14] Karagiannis, Altintas, Ekici: Vehicular Networking: A Survey and Tutorial on Requirements, Architectures, Challenges, Standards and Solutions. IEEE Communications Surveys \& Tutorials, 584-616, 2011.

[15] Buchenscheit, Schaub, Kargl: A VANET-based emergency vehicle warning system. In: IEEE Vehicular Networking Conference, pp. 1-8, 2009.

[16] Kenney, J.B.: Dedicated Short-Range Communications (DSRC) Standards in the United States. Proceedings of the IEEE, 1162-1182, 2011.

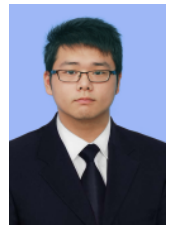

Yu Wang was born in Anhui Province, China, on June 19, 1991. He received his B.E degree in Communication Engineering from Anhui Agriculture University (China) in 2013. He is currently working toward Master's degree at Hefei University of Technology. His major interests are mobile networks, internet of things and computer system.

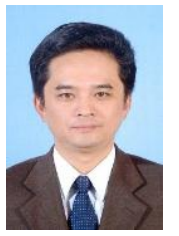

Zhizhong Ding received his B.E degree in Radio Communications from Nanjing University of Aeronautics and Astronautics (China), Master's degree in Circuit and System from Hefei University of Technology (China), and $\mathrm{Ph} . \mathrm{D}$. in Information and Communication Engineering from University of Science and Technology of China. He is currently a Professor with the Department of Communication Engineering, Hefei University of Technology. His research interests include wireless communications, network communications and information theory.

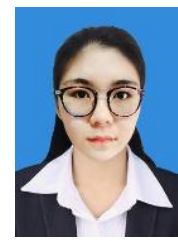

Xue Xia was born in Anhui Province, China, on August 20, 1994. She received her B.E degree in Electronic Information Engineering from Hefei University. She is currently a postgraduate student at Hefei University of Technology. Her major interests are wireless communication and Ultra - high - speed mobile communication

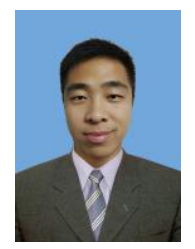

Zhentao Li was born in Anhui Province, China, on November 14, 1993. He received his B.E degree in Communication Engineering from Anhui Normal University, Anhui province, China, in 2015. He is currently working toward Master's degree at Hefei University of Technology, Hefei, China. His major interest is wireless communication.

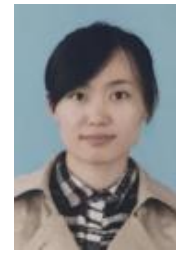

Huijing Shi, Engineer, was born in Shandong Province, China, On November 16,1986. She received her Master' $\mathrm{s}$ degree in Communication and Information System from HeFei University of Technology (China) in 2012. She is currently working in Kunming shipborne equipment research and testing center, engaged in ground support equipment and launch control. 